\section{DYSTROPHINOPATHIES: ANALYSIS OF A COHORT OF 97 PATIENTS}

M. Costa1 ${ }^{1}$, M. Rodrigues ${ }^{1}$, A.R. Gonçalves ${ }^{2}$, R. Santos ${ }^{2}$, M. Santos ${ }^{1}$

${ }^{1}$ Neuromuscular Diseases Consultation, Neuropaediatrics Department, Hospital Maria

Pia, ${ }^{2}$ Genetic Molecular Unit, Center of Medical

Genetics Jacinto Magalhães, INSA, Porto, Portugal

Background: The Dystrophinophaties have two main phenotypes: Duchenne Muscular Dystrophy (DMD) and Becker Muscular Dystrophy (DMB). They are originated in defects of the dystrophin gene.

Objectives: To analyze and characterize a cohort of Dystrophinophaty patients.

Methods: Longitudinal descriptive study (1995 to $2010)$ of Dystrophinophaty patients $(n=97)$ through the consultation of clinical processes.

Results: Eighteen patients had DMB $(3 q)$ and seventy-nine DMD (1우).

Ten patients $(56 \%)$ with DMB had deletions, three $(17 \%)$ duplications, two (11\%) punctual mutations, in one $(5 \%)$ the molecular study was negative and in two $(11 \%)$ it was not accomplished. Of the DMD patients, forty-two $(54 \%)$ had deletions, sixteen (20\%) punctual mutations (two with nonsense mutations), nine (11\%) duplications, in seven (9\%) the molecular study was negative and in five $(6 \%)$ it was not accomplished.

On average first symptoms in DMD were detected at three years and four months. The average age at diagnosis was five years. Loss of motion was on average at nine years and eight months. Heart malfunction was detected on average at twelve years. On average, scoliosis was detected at eleven years and nine months and nocturnal ventilatory support was initiated at fifteen years and eleven months.

Average age of death in DMD patients was eighteen years and eleven months, not being possible to determine the cause of death in the majority of cases.

Conclusions: Detailed characterization at the molecular level of these patients is essential to enable identification of possible candidates for new therapies directed towards the primary genetic defect.

682

\section{SPINAL MUSCULAR ATROPHY IN CHILDREN}

J. Paprocka ${ }^{1}$, E. Jamroz ${ }^{1}$, J. Gawryluk ${ }^{2}$

${ }^{1}$ Child Neurology Department, 2 Student's Scientific Society, Child Neurology Department, Silesian Medical University, Katowice, Poland

Introduction: Spinal muscular atrophy (SMA) is a disease inherited as an autosomal recessive trait, the incidence in Poland is estimated at 1:9319 births. The two genes associated with SMA are SMN1 (survival motor neuron 1gene) and SMN2. About 95-98\% of individuals with SMA are homozygous for the absence of exons 7 and 8 of SMN1 and about $2-5 \%$ are compound heterozygotes for absence of exons 7 and 8 of SMN1 and a point mutation in SMN1.

The aim of this study was to analyze the clinical picture and results of additional studies of patients with SMA.

Material and methods: In the years 2004-2009 in 30 children aged from 6 weeks to 17 years $(\mathrm{M}: \mathrm{F}=16: 14)$ was diagnosed with spinal muscular atrophy (SMA1 N=12, 40\%; SMA2 N=8, 26.7\%; SMA3 $N=10,33.3 \%$ ). In the siblings of three children SMA was confirmed.

Results: Clinical data, in respect to different SMA types, were analyzed. EMG study was abnormal in 27 patients $(90 \%)$, in three children normal results were seen. Nerve conduction study showed reduction of response amplitude in 18 patients $(60 \%)$ and slowing of the conduction velocity in 7 children (23.3\%). All patients underwent molecular study confirming a telomeric deletion of exon 7 in the SMN1 gene.

Conclusions: Based on the presented patients, the most important in the diagnosis of SMA is the clinical presentation and molecular study. Given the clinical symptoms in three patients, despite of normal EMG studies, were performed genetic tests obtaining molecular confirmation of suspected SMA. 